\title{
Systematically dissecting the global mechanism of miRNA functions in mouse pluripotent stem cells
}

\author{
Anyou Wang ${ }^{1 *}$, Qianchuan $\mathrm{He}^{2^{*}}$ and Yan Zhong ${ }^{3}$
}

\begin{abstract}
:
Background: MicroRNAs (miRNAs) critically modulate stem cell properties like pluripotency, but the fundamental mechanism remains largely unknown.

Method: This study systematically analyzes multiple-omics data and builds a systems physical network including genome-wide interactions between miRNAs and their targets to reveal the systems mechanism of miRNA functions in mouse pluripotent stem cells.

Results: Globally, miRNAs directly repress the pluripotent core factors during differentiation state. Surprisingly, during the pluripotent state, the top important miRNAs do not directly regulate the pluripotent core factors as previously thought, but they only directly target the pluripotent signal pathways and directly repress developmental processes. Furthermore, at the pluripotent state miRNAs predominately repress DNA methyltransferases, the core enzymes for DNA methylation. The decreasing methylation repressed by miRNAs in turn activates the top miRNAs and pluripotent core factors, creating an active circuit system to modulate pluripotency.

Conclusion: MiRNAs vary their functions with stem cell states. While miRNAs directly repress pluripotent core factors to facilitate differentiation during the differentiation state, they also help stem cells to maintain pluripotency by activating pluripotent cores through directly repressing DNA methylation systems and primarily inhibiting development in the pluripotent state.
\end{abstract}

Key words: miRNAs, Global mechanism, Stem cells, Systems network, Pluripotency, Functions

\section{Background}

MicroRNAs (miRNAs), short ( 22 nts) conserved endogenous non-coding RNAs, inhibit messenger RNA targets by repressing translation or reducing mRNA stability [1]. MiRNAs critically modulate many cellular events, including the balance between proliferation and differentiation during organ development [1]. In pluripotent stem cells (including induced pluripotent stem cells and embryonic stem cells, referred to as stem cells hereafter), miRNAs play important roles in regulating stem cell bioprocesses [2-6].

miRNAs modulate stem cell pluripotency and differentiation [2-4]. Knocking out the key miRNA processing

\footnotetext{
* Correspondence: anyou.wang@alumni.ucr.edu; qhe@fhcrc.org

${ }^{1}$ School of medicine, University of California San Francisco, S-1268, Medical Sciences Building, 513 Parnassus Ave, San Francisco, CA 94143, USA

${ }^{2}$ Public Health Sciences Division, Fred Hutchinson Cancer Research Center, Seattle, WA 98109, USA

Full list of author information is available at the end of the article
}

enzymes Dicer [2, 3] or DGCR8 [4] causes stem cells to lose their pluripotency. MiRNA-290 cluster has been proposed to regulate the core pluripotency factors like Pou5f1 [7-9]. MiRNA-302-367 cluster has also been used to induce pluripotency [10]. On the other hand, miRNAs like let-7 induce stem cell differentiation [11]. However, these recent studies have mostly focused on individual gene functions in stem cells although genome-wide data might be employed, and the conclusions drawn from these current studies are unavoidably biased on genes selected by these studies. Therefore, these studies only provide partial mechanisms of miRNA functions in stem cells, and the overall systems mechanisms of how miRNAs regulate stem cell processes remain largely elusive.

MiRNAs generally do not work alone to perform their functions [12]. One miRNA might target more than 100

\section{Biomed Central}


genes $[13,14]$, and one gene can be repressed by multiple miRNAs in a sequence-specific fashion [12, 13, 15]. In turn, proteins can physically bind to the promoters and enhancers of miRNAs to regulate miRNA activations [16]. These binary interactions between miRNAs and proteins would form a complex systematic network. This complexity of miRNA interaction network presents a challenge for conventional approaches like geneknockout to unbiasedly capture the real mechanisms of miRNA functions in stem cells.

This present study employed systems physical network approaches [17] and constructed a comprehensive and unbiased map of genome-wide interactions between miRNAs and their targets to investigate the global basis of miRNA roles in pluripotent stem cells, from the pluripotent self-renewal state to the differentiation state. Results of the present study lay a conceptual framework for future studies and applications of miRNAs in stem cells.

\section{Results}

\section{Physical network of miRNA and protein interactions in} stem cells

To systematically reveal the roles of miRNAs in stem cells, this study first constructed a systems network [17] of interactions between miRNAs and proteins by combining multiple published datasets. These interactions contain binary interactions from two directions, from miRNAs to mRNAs coding for proteins, and from proteins to miRNA promoters and enhancers. The miRNA-targets were inferred from CLIP-seq data, which simultaneously identify miRNAs-mRNA interactions by measuring miRNA-Argonaute complexes $[18,19]$ (materials and methods). Protein-targets were inferred from ChIP-seq, which measures protein interactions with DNA [20] (Fig. 1, Additional file 1: Table S1 and materials and methods). The CLIP-seq and ChIP-seq provide data of physical binding interactions. The systems interaction network constructed here includes physical interactions of both miRNA-targets and protein-targets.

The entire network contains $\sim 10,000$ nodes and $\sim 233,000$ interactions (Fig. 1c). Both ChIP-seq and CLIP-seq measures genome-wide targets and thus this network provides a global map of miRNA targets in stem cells. For example, the genome-wide interactions between miRNAs and the pluripotent core factors (Nanog, Pouf1 and Sox2) could be extracted from this network (Fig. 1d). Due to its natural interactions generated from experimental data, this network would provide accurate interactions between miRNA and their targets, and results generated from this network would be reliable.

\section{The primary role of miRNAs in stem cells}

To understand the primary role of all miRNAs activated (up- or down-regulated when compared with somatic cells) at the pluripotent state in stem cells, we searched for the biological functions of the network activated by miRNAs in stem cells. To avoid the dataset biases and to ensure our results general, we included different datasets of miRNAs and genes coding for proteins and selected miRNAs and genes that are only activated with high frequency in all datasets (Additional file 1: Table S2-S3, materials and methods). These activated miRNAs and genes were used to enrich the entire network (Fig. 1) to get the activated network using methods as previously described [17]. The network activated by overexpressed miRNAs and down-regulated proteins (Additional file 1: Table S2-S3) formed a subnetwork activated by up-regulated miRNAs in the pluripotent state. To avoid bias, we run the GO (gene ontology) functional analysis (www.geneontology.org/) of this subnetwork [21] by separately using references of both entire GO annotation database and a set of all up-regulated genes in stem cells. Different references generated different enrichment $\mathrm{p}$-value but produced the similar result that the entire subnetwork primarily functions for development, with corrected p-value $<6.517 \mathrm{e}-62$ (Bonferroni correction using entire annotation, referred as corr, hereafter) (Additional file 1: Figure S1A). This activated subnetwork was further enriched by the developmental GO term to obtain the developmental module (Fig. 2a). This developmental module was decomposed into functional modules based on network topology [22] and it contained 6 sub-functional modules (Materials and Methods). All of these 6 modules primarily function for development (corr p-value $<8.2615 \mathrm{E}-83$, Fig. 2a), indicating that the primary function of over-expressed miRNAs at pluripotent state is to repress developmental modules.

The repressing role of up-regulated miRNAs was further evidenced by examining the targets of three primarily represented miRNA groups, the top overexpressed miRNA group, a miR-302-367 cluster, and a single miR-294. First, a total of 17 out of the 20 most important miRNAs, which were selected on the basis of the variance contribution to the system (material and methods, Additional file 1: Table S4), directly target a developmental sub-network (corr $\mathrm{p}<6.3512 \mathrm{E}-20$, Additional file 1: Figure S2). Among the 17 miRNAs, the top 5 miRNAs also target a module that primarily functions for development (corr $\mathrm{p}<8.5158 \mathrm{E}-23$, Fig. 2b-2c). Furthermore, the well-known miR-302 cluster and even a single miRNA, miR-294, also target modules functionally enriched in the developmental category with respectively corr p-value $<1.4436 \mathrm{E}-21$ (Fig. 3a-3b), and corr p-value $<1.0157 \mathrm{E}-29$ (Fig. 3c-3d). Therefore, overexpressed miRNAs at pluripotent state primarily repress development. Biologically, to maintain the selfrenewal and pluripotency, stem cells have some ways to 


\section{A.miRNA and protein interactions ChIP-seq

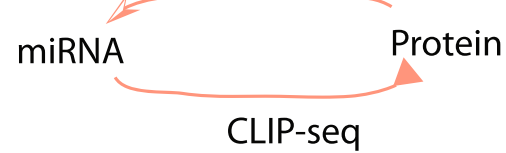 \\ B. Workflow}

Network construction (CLIP-seq, ChIP-seq)

Enriched by expression data (RNA-seq,microarray)

Network dissections of miRNA regulatory mechanisms

Activated miRNAs modulated by DNA methylation (Next-Gen bisulfite sequencing)

\section{A section of entire network}

\section{C.Entire network}

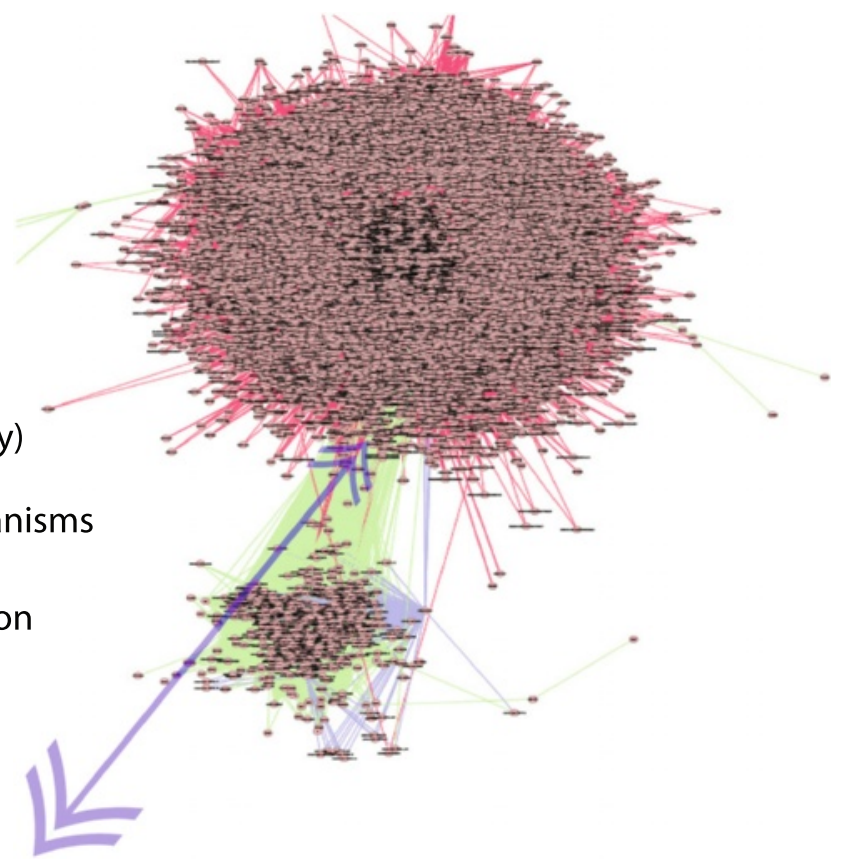

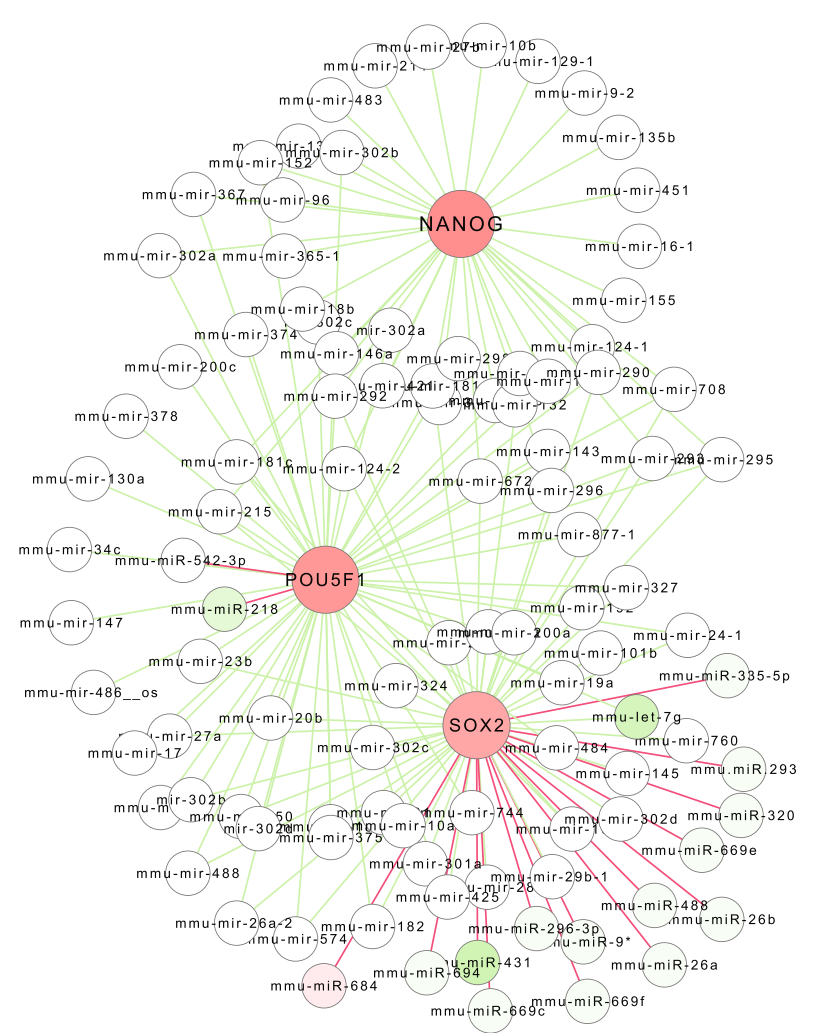

Fig. 1 Overall view. $\mathbf{a}$, The concept of miRNA and protein interactions. $\mathbf{b}$, Workflow of this study. c, Overall view of the entire network constructed by this study. d, A sample of entire network contents shows direct interactions between miRNAs and pluripotent core factors (Nanog, Pou1f5, and Sox2). Nodes denote miRNAs or proteins as labeled; red node represents the gene up-regulation in pluripotent stem cells, green node as down-regulation, and white node as insignificant expression. Edges (lines) represent interactions; red edge denotes miRNAs targeting proteins (from miRNAs to proteins) and green edge represents the binding of proteins with the regulatory elements of miRNAs (from proteins to miRNAs). This labeling strategy applies to all Fig.s in this study 


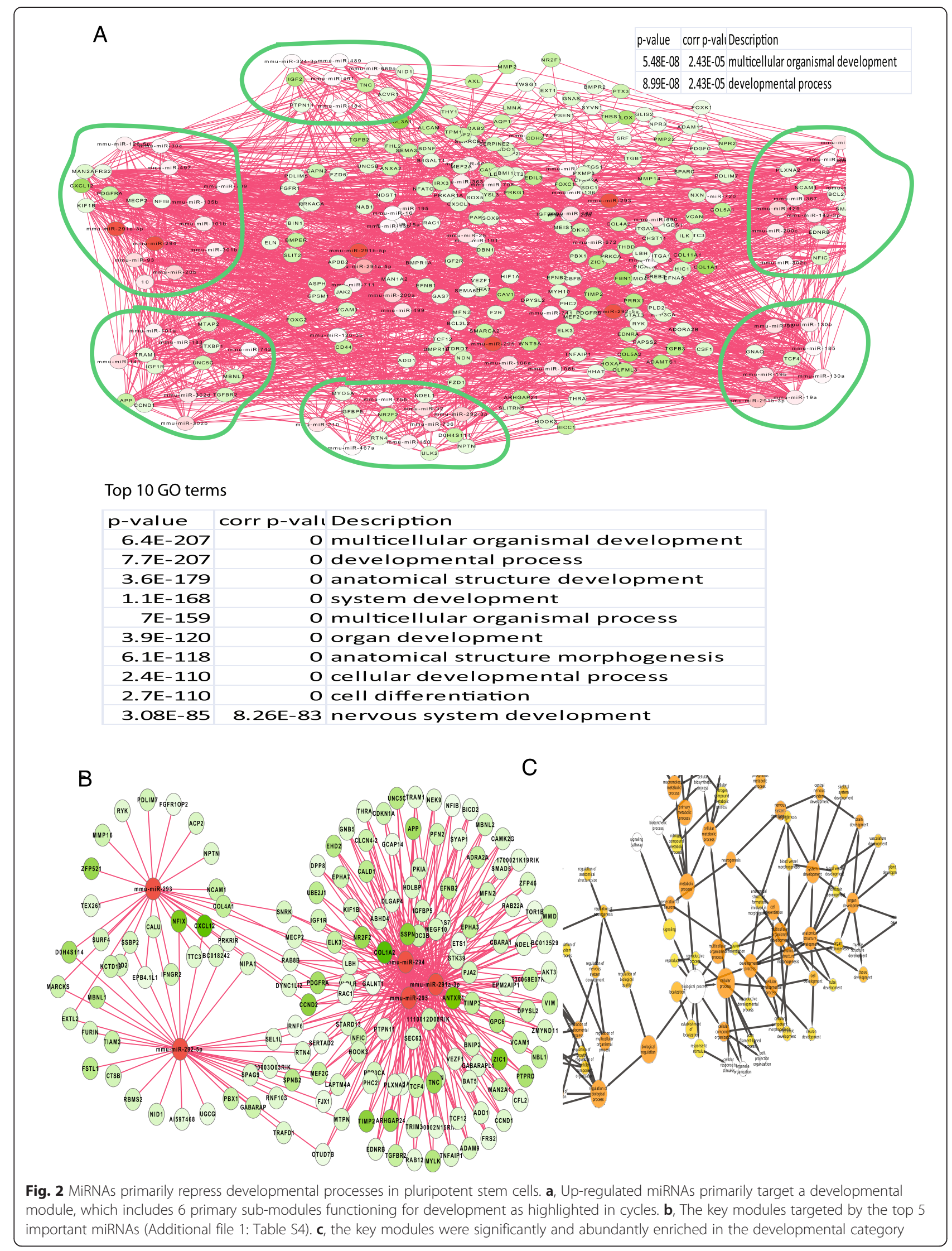




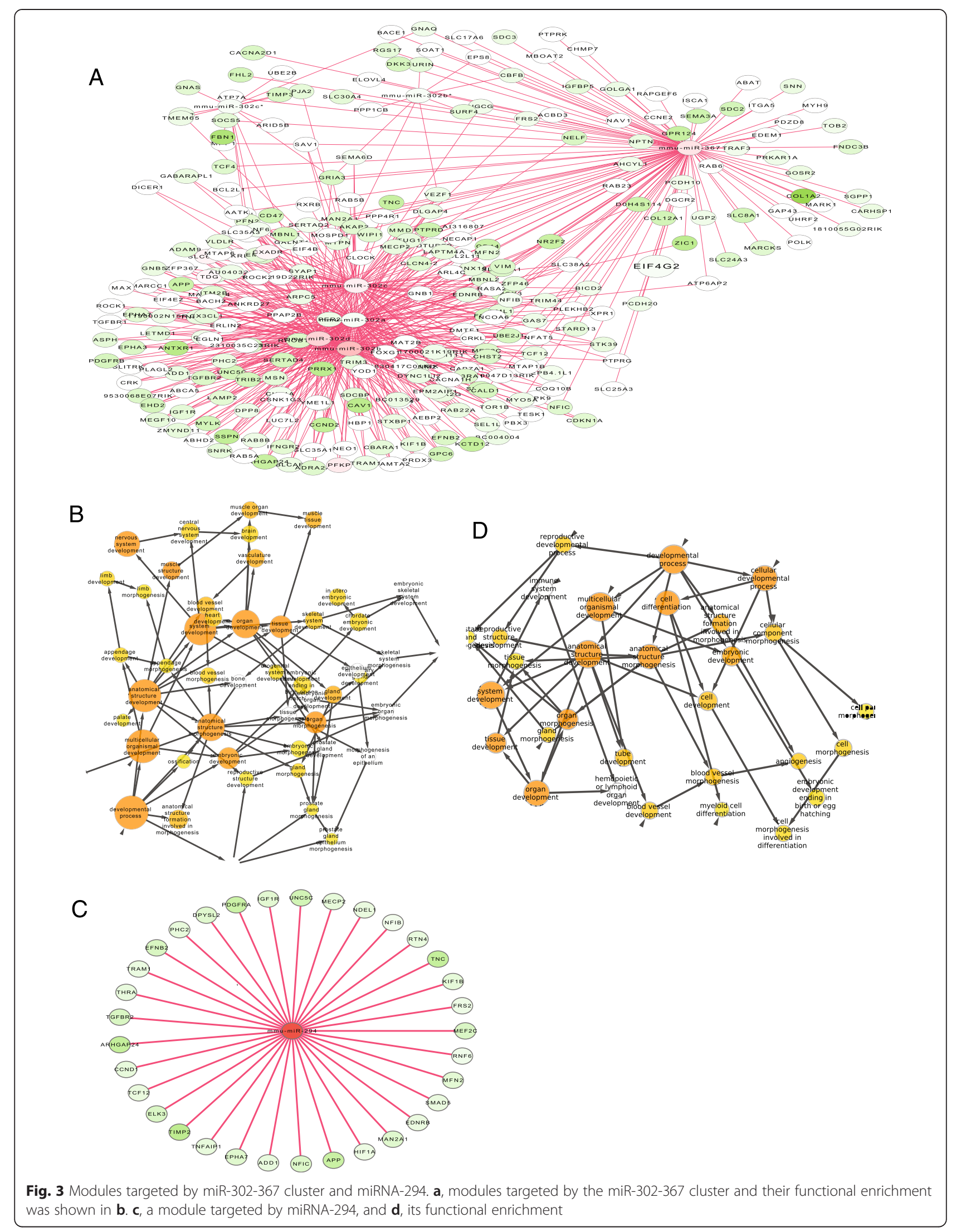


prevent development and differentiation. This repressing function of miRNAs can help miRNAs claim their contributions to the stem cell properties at the pluripotent state.

On the other hand, the down-regulated miRNAs in stem cells directly target genes that primarily function for metabolism and pluripotency, such as Sox2 and Pou5f1 (corr-p < 3.5159E-42, Additional file 1: Figure S1B, Figure S3). These down-regulated miRNAs become upregulated during differentiation and directly slower the entire metabolic processes and inhibit pluripotency during this state. Together, miRNAs primarily and directly repress development during the pluripotent state while they repress metabolism and pluripotency during cell differentiation.

\section{Paths from activated miRNAs to pluripotent core factors} MiRNAs like miR-302 cluster mediate pluripotency $[7,23]$, and it thus was assumed that top over-expressed miRNAs such as miR-302 and miRNA-290-295 cluster in stem cells might directly or indirectly target pluripotent core factors [7, 23]. To investigate if these top miRNAs directly target the core factors, we systematically searched the shortest paths respectively from the top important miRNAs as described above (Materials and Methods, Additional file 1: Table S4) to the three core factors (Pou5f1, Nanog, and Sox2). Surprisingly, all these top miRNAs, including miR-302b, miR-367, miR294, and miR-292, do not directly target any core factors (Fig. 4a-4d). Actually, the direct basis of these miRNAs regulating the pluripotent core factors seemed blurred because all these miRNAs must go through at least 2 steps to reach any pluripotency core factor. These two steps include a miRNA and a protein, which are not consistently expressed with these miRNAs. This suggested that these top over-expressed miRNAs in stem cells do not directly mediate the pluripotency.

To circumvent the bias of selected miRNA groups, we further globally and unbiasedly searched for all upregulated miRNAs that target the pluripotent core factors (Nanog, Pou5f1 and Sox2). Surprisingly, we only found one miRNA (miR-684) that barely up-regulated ( 2 fold changed) [18, 24] (GEO database, methods and materials) in the pluripotent state and directly binds to Sox2 (Fig. 4e), which was also targeted by down-regulated miRNA-431. This indicated that activated miRNAs (>100miRNAs) do not primarily and directly target the pluripotent core factors during the pluripotent state.

On the other hand, regardless of expression, only limited miRNAs directly bind to Pou5f1, while many miRNAs directly target Sox 2 although they might not over-express in stem cells (Fig. 1d), indicating that miRNAs target Sox 2 more than Pou5f1. This suggested that the connection from miRNAs to the pluripotent core factors primarily go through Sox2, in contrast with the current thought that miRNAs should primarily target Pou5f1 [10], a key factor for reprogramming induced pluripotent stem cells.

\section{Pluripotent network targeted by activated miRNAs}

We next expanded the pluripotent gene list to all pluripotent genes uncovered by $\mathrm{Hu}$ et al. [25]. We still focused on the direct miRNA target and searched the first neighbor of all overexpressed miRNAs (Additional file 1: Figure S4A) and all down-regulated miRNAs (Additional file 1: Figure S4B). Most of these targets are shared by up- and down-regulated miRNAs (Fig. 5a-5b), indicating that the primary pluripotent genes in stem cells are carefully modulated by multiple up- and down-regulated miRNAs. The function of the entire shared network is primarily for extrinsic signal pathways associated with pluripotency (Fig. 5b). For example, the highly connected nodes Apc, Rad21 and Eif4g2 are involved in Wnt signaling and mitotic cell cycle pathways $[26,27]$ (corr $=0.017891$, geneontology.org). Signaling pathways with similar functions were also found in the network targeted by over-expressed miRNAs only (Fig. 5c) and in modules directly targeted by the represented miRNAs cluster in stem cells, such as miR-302-367 cluster (Fig. 5d) and miR-294 (Fig. 5e). This indicated that miRNAs in the pluripotent state primarily function for modulating the balance of pluripotent signal pathways instead of directly targeting pluripotent core factors. This suggests that these regulations driven by miRNAs might go through multiple steps to the pluripotent core factors.

Interestingly, Il6st (Interleukin 6 signal transducer, gp130) were regulated by multiple miRNAs in pluripotent state (Fig. $5 \mathrm{~d}-\mathrm{e}$ ). A protein complex constructed by IL6st (gp130) and Lifr (leukemia inhibitory factor receptor alpha) and stabilized by LIF (Leukaemia inhibitory factor) is a regulatory complex to maintain self-renewal in stem cells [28]. Targeting Il6st here further suggested the important role of miRNAs in modulating pluripotency.

In contrast to the up-regulated miRNAs in pluripotent state, down-regulated miRNAs directly target the core pluripotency factors like miRNA431 targeting Sox2 (Additional file 1: Figure S5). These down-regulated miRNAs in the pluripotent state are actually upregulated during the differentiation state and these pluripotency core factors become down-regulated in differentiation. This direct linkage between up-regulated miRNAs and down-regulated core factors suggested that these miRNAs directly inhibit the pluripotent core factors and repress pluripotency to facilitate differentiation during stem cell differentiation. This result parallels with recent experimental observations showing that miRNAs repress 


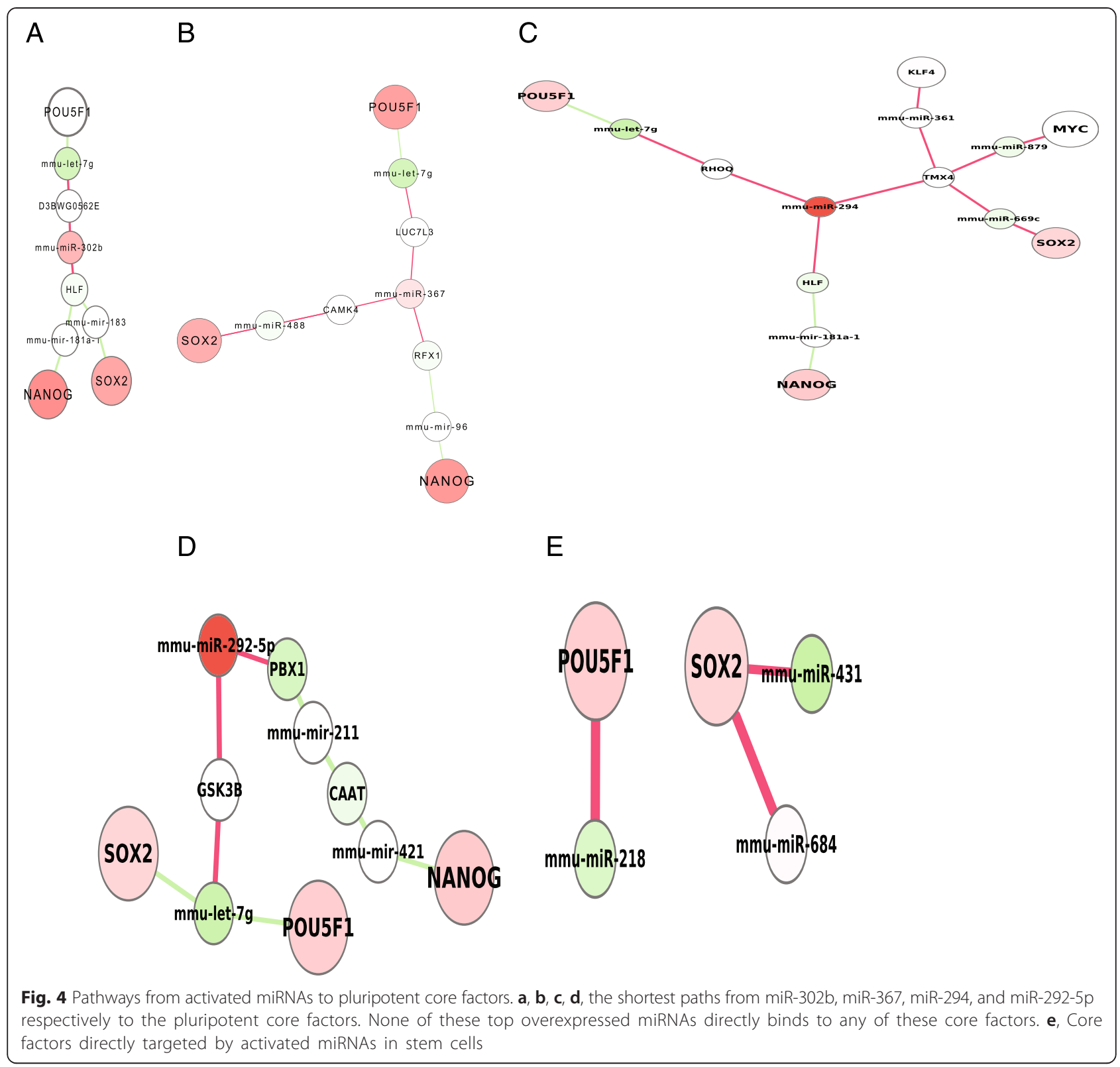

the pluripotent core factors and pluripotency during differentiation [16].

To summarize, miRNAs do not directly target pluripotent core factors during pluripotent state but miRNAs directly target and repress these core factors during differentiation.

\section{MiRNAs abundantly target epigenetic system}

The above result indicated that the number of miRNA binding (degree) to pluripotent genes (nodes) was very limited. The target with the highest degree, Eif4g2, was only attacked by $\sim 20$ up-regulated miRNAs (Fig. $5 \mathrm{c}$ ). It was expected that a certain group of nodes should be targeted by more than that. This drove us to further search the network hubs (the important nodes) in the entire network. We systematically ranked the miRNA targets by degree (miRNA directly binding only) and obtained the top hubs. The top hubs mostly function for RNA processing, but surprisingly, Dnmt3a, a DNAmethyltransferase for de novo DNA methylation, was among the top hubs. Dnmt3a actually holds more than 160 miRNA binding sites in 3'-UTR region based only on 8 bp seed mapping and it was ranked within the top $1 \%$ of the up-regulated miRNA targets (Fig. 6a). Dnmt3a was even ranked higher than Eif4g2, the highest ranked node in the pluripotent genes (Fig. 6a, Fig. 5b-c), indicating that Dnmt3a should be a top important node in the network directly regulated by miRNAs in the 


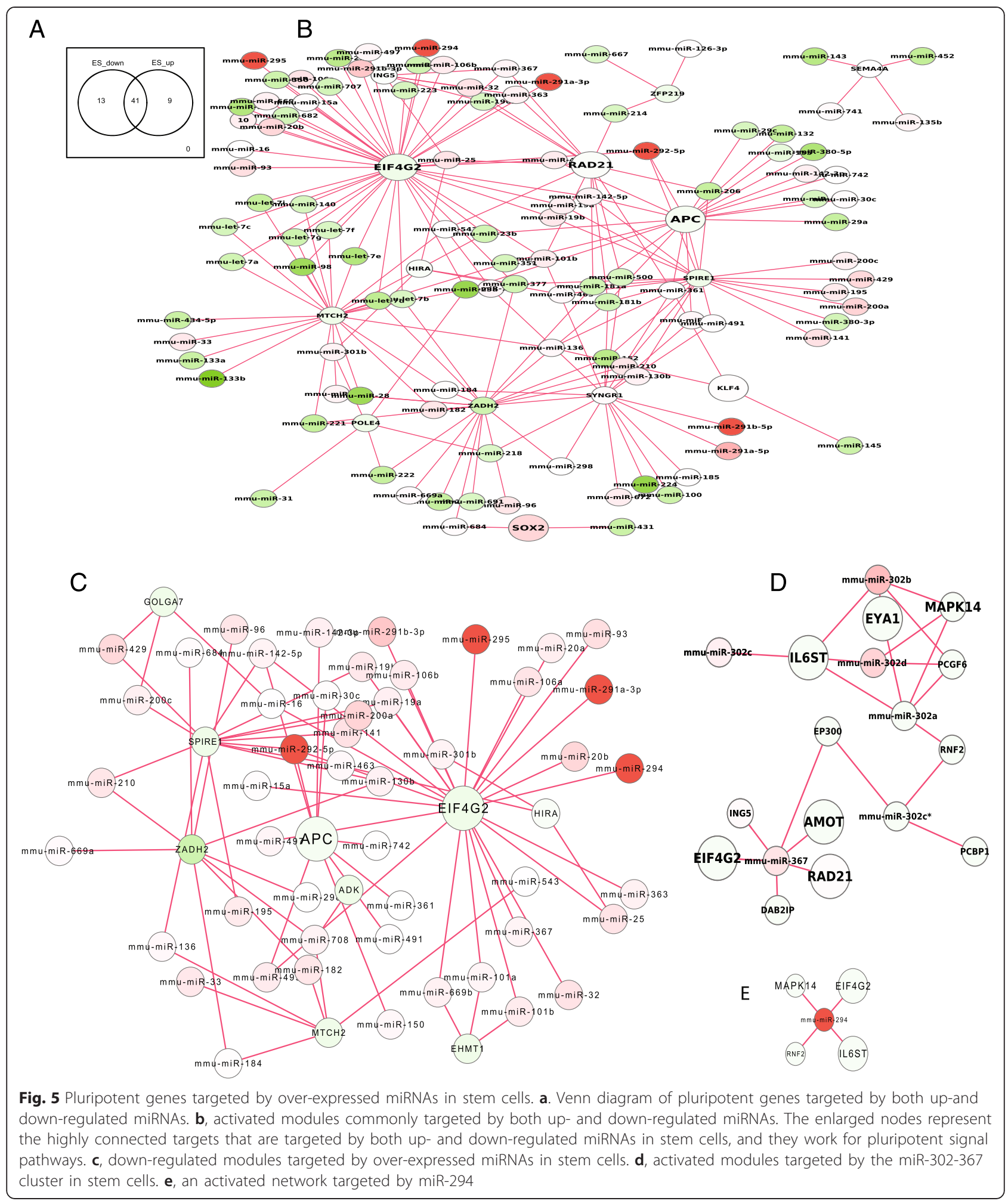

pluripotent state. This also indicated that miRNAs predominately target DNA methylation system, rather than the pluripotent genes. We extracted the network of Dnmt3a directly targeted by miRNAs and found that the top miRNA clusters (Additional file 1: Table S4), such as miR-302-367 and miR-290-295 cluster, were among the miRNAs that target Dnmt3a (Fig. 6b). Similarly, Many well-known miRNA clusters (e.g. miR-290-295 
A

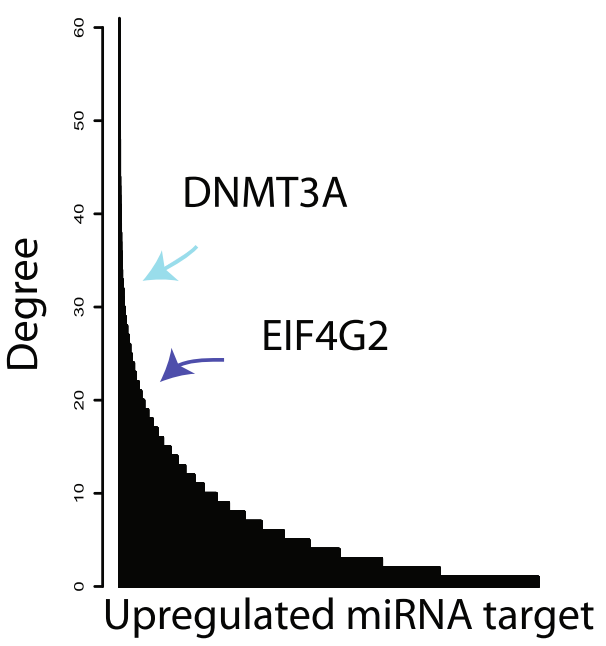

B
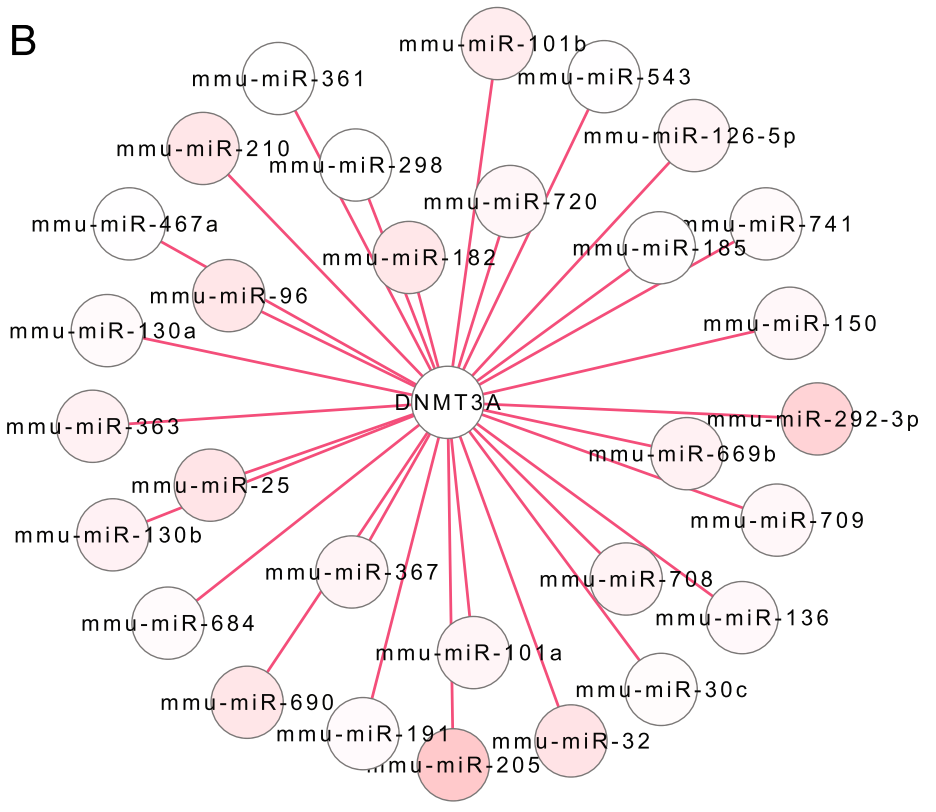

C

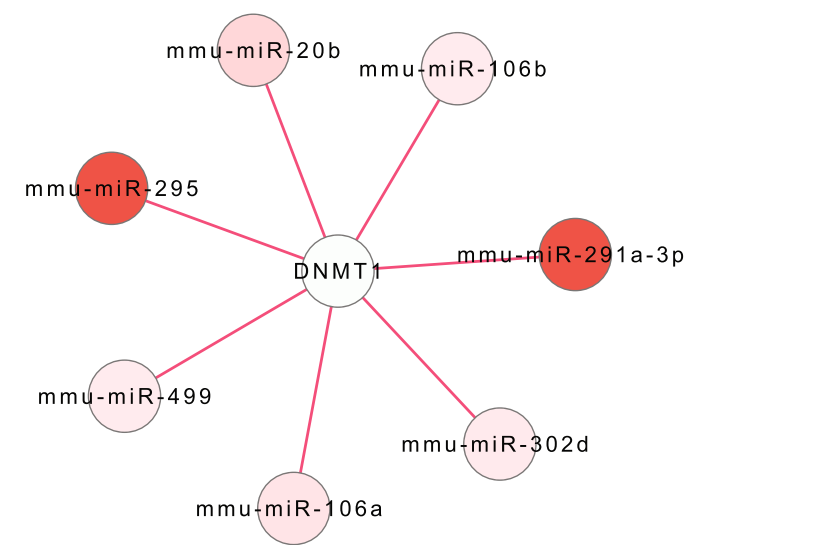

Fig. 6 (See legend on next page.) 
(See figure on previous page.)

Fig. 6 MiRNAs abundantly target DNA methylation systems. a, Dnmt3a was targeted by 30 up-regulated miRNAs and was ranked in the top $1 \%$ of the up-regulated miRNA targets. The network nodes (miRNA targets) were plotted against the node degree (miRNA binding number only). b, the Dnmt3a network targeted by miR NAs. The most important miRNAs, including the miR-302-367 and miR-290-295 cluster, are found in the Dnmt3a network. c, miRNAs target another methylation enzyme Dnmt1

and miR-302) in stem cells also target Dnmt1 (Fig. 6c), an enzyme predominately responsible for methylation in hemimethylated $\mathrm{CpG}$ islands. Many down-regulated miRNAs also target Dnmt3a and Dnmt1 (Additional file 1: Figure S6), but their attacks would lead to differentiation instead of maintaining pluripotency in stem cells as discussed above [16]. These abundantly overexpressed miRNAs that target the methylation system suggest that miRNAs predominately repress Dnmts in the pluripotent state.

In addition, miRNAs directly and abundantly target a core histone modification complex (Hdac4-Mef2cMef2d, http://www.ncbi.nlm.nih.gov/gene/9759) (Fig. 7), including Mef2c (myocyte enhancer factor 2C), which was targeted by the top over-expressed miRNA clusters including miR-290-295 and miR-302 cluster (Fig. 7). Upregulating Mef2c enhances stem cells differentiation [29], and down-regulated Mef2c inhibits differentiation [29]. The down-regulation of Mef2c targeted by the top overexpressed miRNA clusters suggests that miRNAs repress differentiation in stem cells. This is consistent with our discussion above on the miRNA repressing development and differentiation at the pluripotent state in stem cells (Figs. 2 and 3). Together, miRNAs directly and abundantly target the epigenetic systems at the pluripotent state.

\section{DNA methylation mediates the miRNA activation in stem cells}

To search the mechanism controlling the miRNA activations, this study turned to the genome-wide sequencing of DNA methylation in stem cells and methylationloss-stem cells [30] (Additional file 1: Table S1, materials and methods). A total of 2000 bp in each upstream and downstream of start sites of all activated miRNAs were examined. While the DNA methylation in the downstream of up-regulated miRNAs is not different from that of down-regulated miRNAs ( $p>0.1899)$, the down-regulated miRNAs hold significantly higher methylation upstream than up-regulated miRNAs $(\mathrm{p}<3.685 \mathrm{e}-05$, Fig. 8a). Surprisingly, the biggest difference locates in $\sim 1000$ bp up-stream instead of immediate upstream ( $\mathrm{p}<1.265 \mathrm{e}-06$, Fig. $8 \mathrm{~b}$ ). Furthermore, these differences are overall negatively correlated to miRNA expressions with correlation coefficient of -0.35 and p-value $<0.05$ (Fig. 8c). This suggested that the difference in DNA methylation accounts for the miRNA activations. This parallels a recent observation showing that the loss of DNA methylation significantly increases miRNA expressions [29]. Therefore, miRNA activations and their network are mediated by DNA methylation in 1000 bp upstream regions.

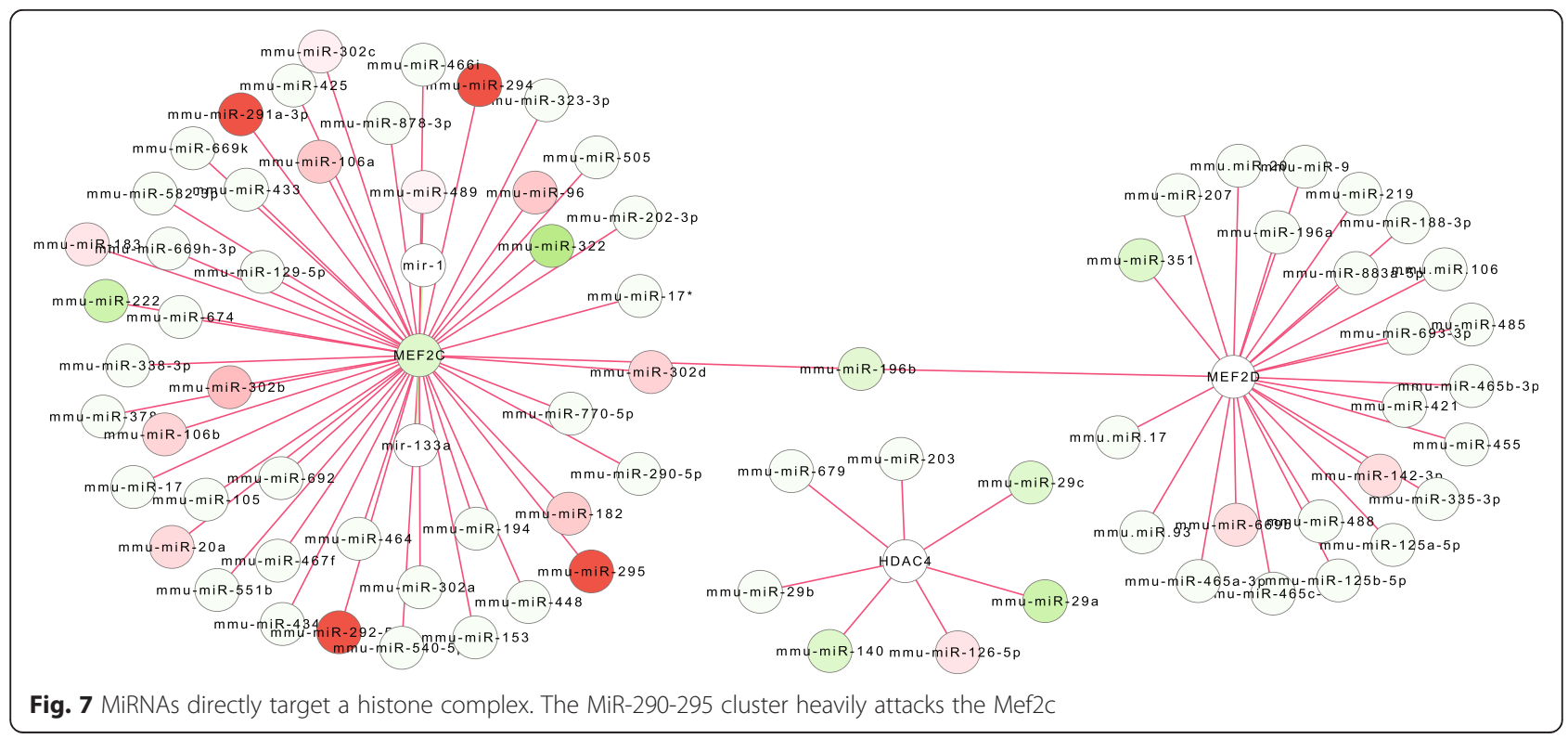



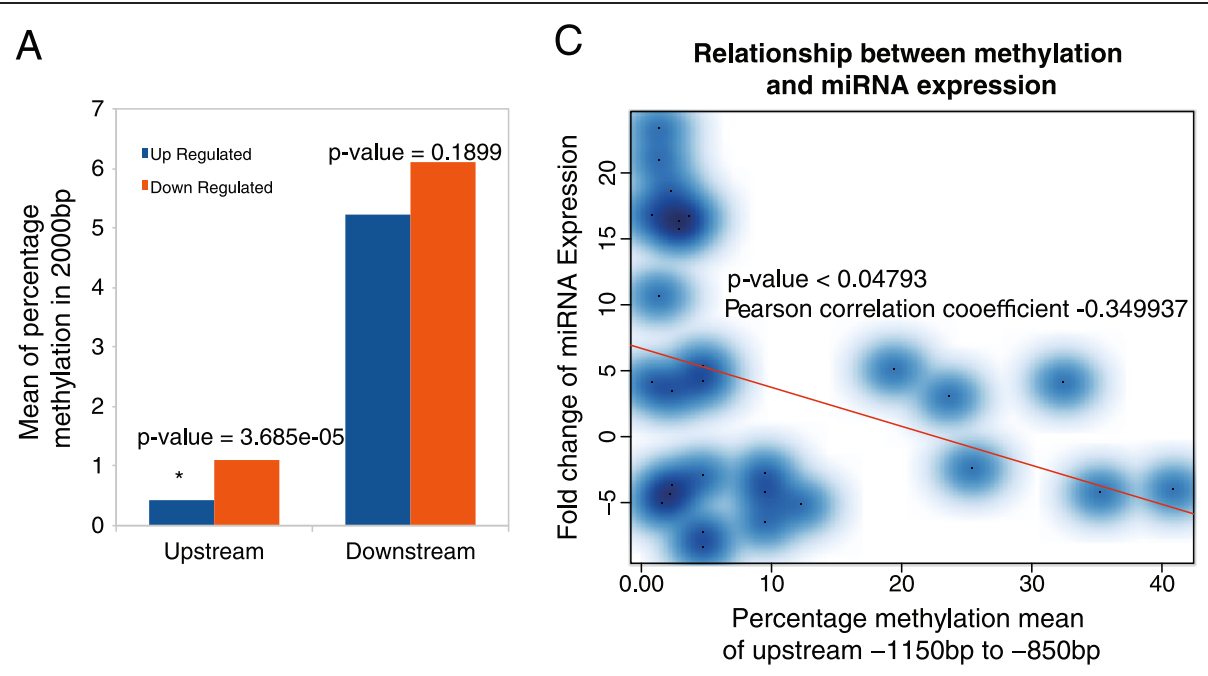

B
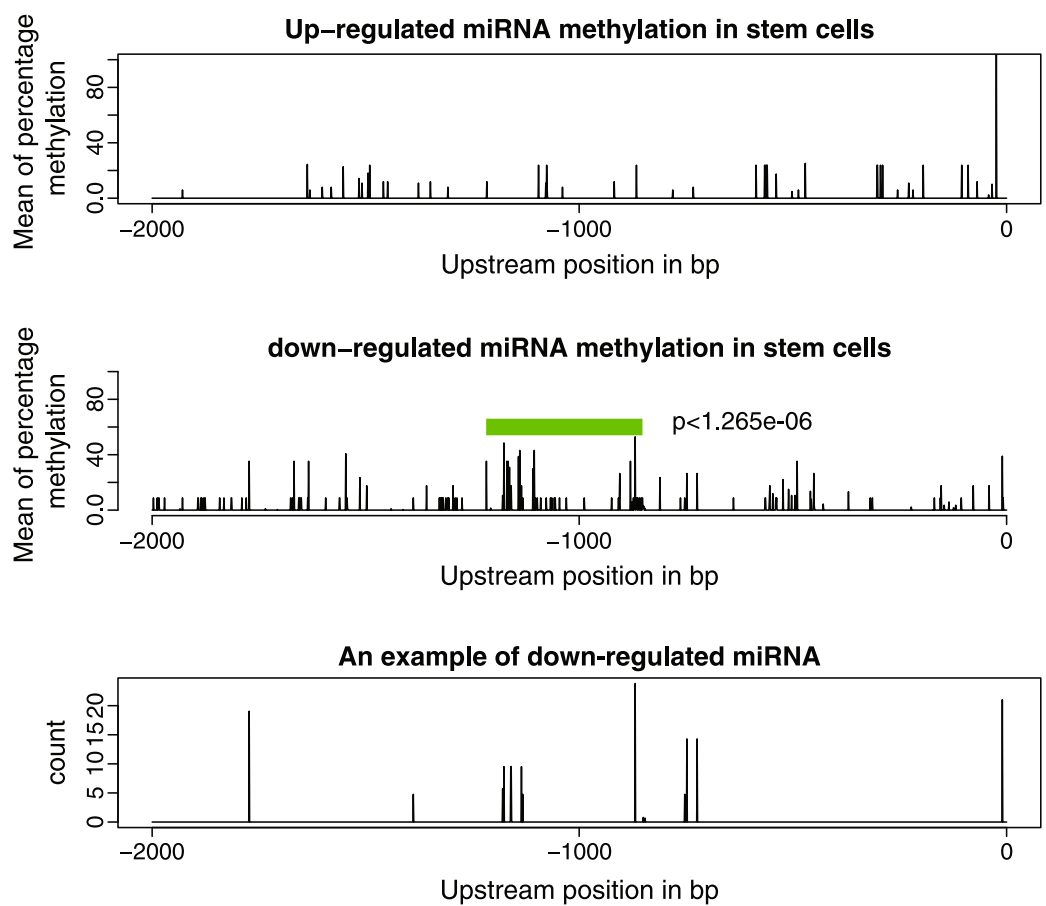

Fig. 8 DNA methylation mediates miRNA activations in stem cells. a, The methylation levels upstream and downstream from the miRNA start site. Down-regulated miRNAs have significantly higher methylation in upstream region when compared with up-regulated miRNAs. $\mathbf{b}$, Detailed methylation profiling for regions that are $2000 \mathrm{bp}$ upstream from the activated miRNAs. The top 30 down-regulated miRNAs (Additional file 1: Table S2) have higher methylation around $-1000 \mathrm{bp}$ (green highlighted in middle panel) than that of the top 30 up-regulated miRNAs (Additional file 1: Table S2, upper panel). The methylation profiling of a single down-regulated miRNA-133 as a representative example (bottom panel). c. Negative correlation between DNA methylation and miRNA expression. Red line represents regression line

\section{Discussion}

This study is the first investigation, to our knowledge, to search the primary mechanism of miRNA functions in stem cells at systems level on the basis of a physical map constructed by direct interactions of miRNAs and proteins. MiRNAs play critical roles in maintaining overall properties like pluripotency in stem cells [2-4], but the mechanisms still remain largely elusive. Here, we systematically revealed that miRNA primarily target developmental modules during the pluripotent state while another set of miRNAs directly target pluripotent core factors during differentiation state. This suggests that one of miRNA primary functions is to repress development at the pluripotent state to prevent stem cell 


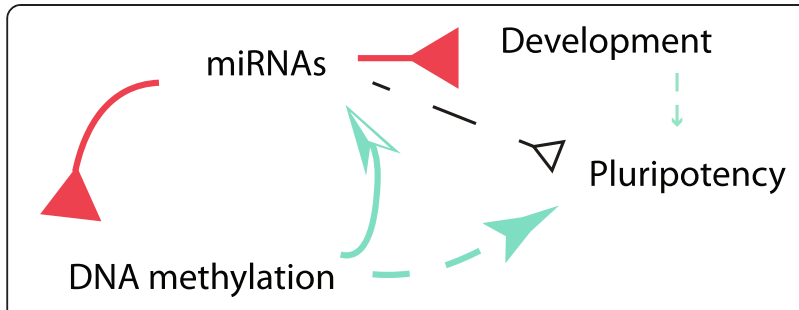

Fig. 9 A system view of miRNAs primary mechanistic roles in maintaining pluripotency in pluripotent stem cells. MiRNAs directly repress both the DNA methylation system and the development, while indirectly regulate pluripotency genes. Reduced DNA methylation activates the miRNAs and pluripotent core factors for pluripotency. The activated circuit between miRNAs and DNA methylation, as well as the development inhibition, help stem cells to maintain the pluripotent state, see text for detail. The solid lines are directly derived from the present study and they represent direct interactions and the dash dark line represents indirect interactions, while dash green lines denote evidences from reference papers.

differentiation and to keep stem cell pluripotency while miRNAs also degrade pluripotent core factors to facilitate differentiation during differentiation state. This is consistent with the recent observation that overexpressions of miRNAs induce pluripotency [10] and miRNAs also facilitate stem cells differentiation [16].

The mechanism of miRNAs in regulating pluripotency has been widely investigated [7-9], but the systems mechanism remains elusive. One of fundamental questions is whether miRNAs directly or indirectly regulate the pluripotency at system level. It has been consistently observed that gene expressions of the top overexpressed miRNAs are positive correlative to that of pluripotent core factors. Recent evidences also show that miRNA-302-367 cluster could induce pluripotency [10]. The observations led to a speculation that miRNAs might directly target the pluripotent core factors. Although miRNAs could have many functions in certain conditions, the primary functions of miRNAs are for degrading and inhibiting their targets. If the overexpressed miRNAs directly target the core factors, these miRNAs would likely repress the core factors as previously evidenced [31], leading to down-regulations of these core factors. A negative correlation between them was expected, but in fact, a positive correlation has been consistently observed. This suggests that the top miRNAs might not directly target and degrade pluripotent core factors. Here, we utilized the power of our system network to exhaustively search the direct linkages between miRNAs and the pluripotent core factors. Our results revealed that the top miRNAs such as miR-290 and miRNA-302 cluster do not directly target any core pluripotent factors during the pluripotent state (Fig. 4). Most of top miRNAs only directly target the extrinsic signal pathways associated with pluripotency (Fig. 5). A signal from a signal pathway normally travels through multiple steps to reach its targets. Therefore, the top miRNAs indirectly target the core factors and indirectly regulate the pluripotency.

In contrast to the indirect mechanism of miRNAs in targeting the genetic system, the top miRNAs, including miR-302-367 and miRNA-294 cluster, directly and abundantly target the core enzymes of DNA methylation system, Dnmt3a and Dnmt1. Targeting these Dnmts by the top expressed miRNAs suggests inhibition of Dnmts, which highly express in somatic cells but lowly express in stem cells. This parallels the most recent observations showing that gene expressions of up-regulated miRNAs are negatively correlative with that of DNA methyltransferases (Dnmts) [32, 33] and that microRNAs degrade Dnmts in stem cells [32].

During self-renewal and pluripotency, mouse stem cell genome are in hypomethylated status, and genome-wide three methylase (Dnmt3a, Dnmt3b, and Dnmt1) are tightly depressed [34]. Our data showed that abundant miRNA directly target DNA methylase, suggesting that miRNAs play a crucial role in repressing DNA methylation machinery during pluripotency.

Our result of miRNA directly repressing Dnmts contributes to the understanding of the positive relationship existing between up-regulated miRNAs and overexpressed core pluripotent factors in stem cells as discussed above. Over-expressed miRNAs directly target Dnmts, leading to DNA methylation reductions at genome-wide level, including the loci of the pluripotent core factors. This results in over-expression of these core factors responsible for pluripotency. This is consistent with the observation of less methylation in the loci of the core factors during induced pluripotent stem cell reprogramming, and it is also consistent with our recent finding that the demethylation level can be used as a variable for discriminating different stem cells [35]. Therefore, miRNAs primarily and directly target the epigenetic system that further activates pluripotent core factors in stem cells. This parallels the most current report that miRNAs degrade Dnmts in stem cells [32].

DNA methylation might regulate expressions of a certain group of genes in stem cells [36]. Our data further showed that DNA methylation globally mediates the miRNA activations in stem cells. These miRNA activations by DNA methylation in turn repress the DNA methylation. Less methylation activates the miRNAs and pluripotent core factors again. Together, we proposed a system-wide circuit to describe a part of miRNA primary roles in modulating pluripotency in pluripotent stem cells (Fig. 9). In this circuit, miRNAs directly repress development and directly repress the DNA methylation system, while miRNAs indirectly regulate pluripotency genes. This repression of DNA methylation activates 
both pluripotent factors and miRNAs. The activations of pluripotent factors and repression of development contribute to pluripotency in stem cells, while the activations of miRNAs further inhibit both DNA methylation and development. This creates an active system-wide circuit in stem cells to maintain the pluripotent state (Fig. 9).

The network interactions of proteins targeted by multiple miRNAs also provide a systems vision of quantitative regulatory mechanism of miRNAs in stem cells. For example, our study uncovered that many up- and down-regulated miRNAs directly target Dnmts and Wnt pathway like Apc, suggesting that DNA methylation and Wnt pathway are delicately regulated in stem cells dynamically. These regulations are controlled by not only simply turning on/off genes but also mediated by the quantitative concentrations of many gene expressions. The slightly quantitative alternation of each component concentration could result in change of stem cell state, self-renewal or differentiation.

This quantitative regulatory mechanism can help to revise the controversial mechanisms derived from individual gene studies. For example, Wnt signaling and pathway components interact with pluripotent core factors (Oct4/ SoX2/Nanog) to regulate stem cell pluripotency and differentiation [26, 27], but whether Wnt signaling promotes pluripotency or differentiation has been intensively debated recently [26, 27], partially due to lack of system-wide vision on it. These components targeted by miRNAs revealed here with other system-network level data could provide novel clues to design biological experiments and select mathematical models to clarify the role of Wnt pathway in stem cells and to finally solve this controversial issue.

\section{Conclusion}

This study mostly focused on the primary functions of miRNAs in pluripotent state. We systematically revealed that miRNAs directly repress DNA methylation systems and primarily inhibiting development to help stem cells to maintain pluripotency in the pluripotent state. However, we do not exclude other functions of miRNAs in stem cells. The results made here are based on the current limited data and these miRNA functions drawn here only account for a part of miRNA roles in stem cells. With data accumulating, more functions of miRNAs will certainly be explored. Recently, an exciting field of noncoding RNA studies have revealed that long noncoding RNAs (lncRNAs), transcripts of more than 200 nucleotides, participate in the regulation of the pluripotent state and differentiation. These IncRNAs even regulate the complex miRNA network and interact with DNA methylation machinery like methylase [37]. A more exciting comprehensive map of miRNA-lncRNADNA methylation-mRNA can be expected. Nevertheless, our key results and conclusion regarding the fundamental basis of primary miRNA functions in regulating mouse stem cell pluripotency and differentiation have actually also been observed in human $[10,16]$, suggesting that these miRNA functions might be conserved in both mouse and human. Understanding the fundamental systems roles of miRNAs studied here would broadly direct the future functional studies of miRNAs in both human and mouse stem cells.

\section{Materials and methods Data resources}

This study analyzed multiple datasets published in public database, GEO database (www.ncbi.nlm.nih.gov/geo/). These data were generated by high-throughput methods as detailed in Additional file 1: Table S1, including CLIP-seq, ChIP-seq, RNA-seq, microarray, and bisulfite sequencing DNA (Additional file 1: Table S1).

\section{Data bioinformatics analysis}

To be consistent and comparable, all sequencing data were mapped to mouse genome $(\mathrm{mm} 9)$. All fastq files (Additional file 1: Table S1) from CLIP-seq, ChIP-seq and RNA-seq were aligned using BWA 0.6.2 with default parameters [38]. All PCR-duplicates were removed. Bisulfite sequencing DNA was aligned by Bismark 0.7.6 with tolerating one non-bisulfite mismatch per read (http:// www.bioinformatics.babraham.ac.uk/projects/bismark/).

The clusters from CLIP-seq were generated by using GenomicRanges 1.12 under R 2.15 and were subjected to second noise quartile cutoff. The filtered clusters located in 3'UTRs and CDSs were used to search for miRNA bindings. The miRNA bindings were searched against the perfect match of miRNA seed sequences (6-8 nt) using home-made python scripts. The seed sequences were extracted from miRNA sequences downloaded from miRBase 19 (http://microrna.sanger.ac.uk/). Peaks from ChIP-seq were called using SISSRs 1.4 (http:// dir.nhlbi.nih.gov/papers/lmi/epigenomes/sissrs/) and the peaks were annotated using ChIPpeakAnno 2.8 under $\mathrm{R}-2.15$. The differential expressions from RNA-seq were performed using negative binomial model using edge 3.2 under $R$ 2.15. Bisulfite DNA methylation was counted against the genome coordinates of miRNAs (Additional file 1: Table S4). Microarray data were analyzed using limma 3.16 under R 2.15.

\section{Activated genes and miRNAs selection}

To minimize the biases from individual experiments and cell lines as well as the confounders caused by highthroughput methods, we selected genes and miRNAs activated in different conditions by using different sets of data (Additional file 1: Table S1). Expression data from various resources (Additional file 1: Table S1) were employed. Genes coding for proteins with activating frequency $>50 \%$ in all observations and miRNAs with activating frequency of more than $25 \%$ out of all observations were treated as activated genes and were selected 
(Additional file 1: Table S2-S3). The up-regulation and down-regulation was based on comparison to somatic cells in each experiment (corrected $\mathrm{p}<0.01$ and fold change $>2$ ).

The top important miRNAs (Additional file 1: Table S4) were selected on the basis of their contributions to network structure and variance by using the algorithm as we previously published [36]. Briefly, the top miRNAs were selected on the basis of their ranking scores calculated by the eigengene-based connectivity as defined below [36].

$$
\left.S C O R E=\frac{d_{i}}{d_{\max }}+2 \times \operatorname{cor} \mid X i, E\right) \mid
$$

where $d_{i}$ denotes the $i^{\text {th }}$ node degree, and $d_{\max }$ denotes the maximum degree of a node in the entire network. $|\operatorname{Cor}(\mathrm{xi}, \mathrm{E})|$ represents the absolute Pearson correlation coefficient, where $x_{i}$ represents a vector of $i^{\text {th }}$ node value, and $\mathrm{E}$ eigengene of the network.

\section{Network construction and analysis}

The network construction and analysis were performed by approaches as our previous reports [17, 39]. Briefly, we built the map (Fig. 1) by collecting the interactions of both miRNAs targets from the CLIP-seq and proteinbinding promoters of miRNAs from ChIP-seq data. The interactions from CLIP-seq and ChIP-seq were signed as miRNA or proteins respectively (Fig. 1). Only the direct interactions (first-neighbor) were selected and included, and thus this map is a physical binding network. The network was enriched by the activated genes and miRNAs selected above to get the activated network. Functional modules were further enriched by the functional genes based on gene ontology enrichment (e.g. Additional file 1: Figure S1, http://www.geneontology.org/). Six sub-modules in functional developmental module (Fig. 2a) were based on network topology to identify the densely connected modules. The target node ranking was based on the degree of each miRNA node (Fig. 6a-6b).

\section{Additional file}

Additional file 1: Figure S1. Functional enrichments of all targets that are targeted by all up- and down-regulated miRNAs. A, Up-regulated miRNAs. B, down-regulated miRNAs. Figure S2. Total 17 out of top 20 important miRNAs target developmental genes. Figure S3. Down-regulated miRNAs mediate metabolism in stem cells. Figure S4. All pluripotent genes targeted by up-regulated miRNAs (A) and down-regulated miRNAs (B) in stem cells. Figure S5. An activated network targeted by down-regulated miRNAs. Figure S6. Down-regulated miRNAs targeting DNMT3A. Table S1. Data sources. Table S2. A list of miRNAs that are frequently and significantly differential expressed between stem cells and somatic cells. Table S3. A list of genes coding for proteins that are frequently and significantly differential expressed between stem cells and somatic cells. Table S4. A list of top 20 important miRNAs.

\section{Competing interests}

The authors declare that they have no competing interest.

\section{Author contributions}

A.W, conceived and designed the experiments, analyzed the data, contributed data/materials/analysis tools, wrote the manuscript. Y.Z. contributed data/ materials/analysis tools. Q.H. contributed data/materials/analysis tools/ writing. All authors reviewed the manuscript.

\section{Acknowledgments}

We specially thank Drs. Ying Du and Chunxiao Zhou for data collection.

\section{Author details}

${ }^{1}$ School of medicine, University of California San Francisco, S-1268, Medical Sciences Building, 513 Parnassus Ave, San Francisco, CA 94143, USA. ${ }^{2}$ Public Health Sciences Division, Fred Hutchinson Cancer Research Center, Seattle, WA 98109, USA. ${ }^{3}$ Division of Gynecologic Oncology, Linyi Tumor Hospital, Shandong 276000, China.

Received: 11 August 2014 Accepted: 16 June 2015

Published online: 01 July 2015

\section{References}

1. Kloosterman WP, Plasterk RH. The diverse functions of microRNAs in animal development and disease. Dev Cell. 2006;11(4):441-50.

2. Kanellopoulou C, Muljo SA, Kung AL, Ganesan S, Drapkin R, Jenuwein T, Livingston DM, Rajewsky K. Dicer-deficient mouse embryonic stem cells are defective in differentiation and centromeric silencing. Genes Dev. 2005;19(4):489-501.

3. Murchison EP, Partridge JF, Tam OH, Cheloufi S, Hannon GJ. Characterization of Dicer-deficient murine embryonic stem cells. Proc Natl Acad Sci U S A. 2005;102(34):12135-40.

4. Wang Y, Medvid R, Melton C, Jaenisch R, Blelloch R. DGCR8 is essential for microRNA biogenesis and silencing of embryonic stem cell self-renewal. Nat Genet. 2007;39(3):380-5.

5. Chen CZ, Li L, Lodish HF, Bartel DP. MicroRNAs modulate hematopoietic lineage differentiation. Science. 2004;303(5654):83-6.

6. Cordes KR, Sheehy NT, White MP, Berry EC, Morton SU, Muth AN, Lee TH, Miano JM, Ivey KN, Srivastava D. miR-145 and miR-143 regulate smooth muscle cell fate and plasticity. Nature. 2009;460(7256):705-10.

7. Hanina SA, Mifsud W, Down TA, Hayashi K, O'Carroll D, Lao K, Miska EA, Surani MA. Genome-wide identification of targets and function of individual MicroRNAs in mouse embryonic stem cells. PLoS Genet. 2010;6(10), e1001163.

8. Benetti R, Gonzalo S, Jaco I, Munoz P, Gonzalez S, Schoeftner S, Murchison $E_{\text {, And }} \mathrm{T}$, Chen $\mathrm{T}$, Klatt $\mathrm{P}$, et al. A mammalian microRNA cluster controls DNA methylation and telomere recombination via Rbl2-dependent regulation of DNA methyltransferases. Nat Struct Mol Biol. 2008;15(3):268-79.

9. Sinkkonen L, Hugenschmidt T, Berninger P, Gaidatzis D, Mohn F, Artus-Revel CG, Zavolan M, Svoboda P, Filipowicz W. MicroRNAs control de novo DNA methylation through regulation of transcriptional repressors in mouse embryonic stem cells. Nat Struct Mol Biol. 2008;15(3):259-67.

10. Anokye-Danso F, Trivedi CM, Juhr D, Gupta M, Cui Z, Tian Y, Zhang Y, Yang W, Gruber PJ, Epstein JA, et al. Highly efficient miRNA-mediated reprogramming of mouse and human somatic cells to pluripotency. Cell Stem Cell. 2011;8(4):376-88.

11. Melton C, Judson RL, Blelloch R. Opposing microRNA families regulate selfrenewal in mouse embryonic stem cells. Nature. 2010;463(7281):621-6.

12. Stefani G, Slack FJ. Small non-coding RNAs in animal development. Nat Rev Mol Cell Biol. 2008;9(3):219-30.

13. Bartel DP. MicroRNAs: target recognition and regulatory functions. Cell. 2009;136(2):215-33.

14. Chen C, Ridzon D, Lee CT, Blake J, Sun Y, Strauss WM. Defining embryonic stem cell identity using differentiation-related microRNAs and their potential targets. Mamm Genome. 2007;18(5):316-27.

15. Rajewsky N. microRNA target predictions in animals. Nat Genet. 2006:38(Suppl):S8-S13.

16. Xu N, Papagiannakopoulos T, Pan G, Thomson JA, Kosik KS. MicroRNA-145 regulates $\mathrm{OCT} 4, \mathrm{SOX} 2$, and KLF4 and represses pluripotency in human embryonic stem cells. Cell. 2009;137(4):647-58. 
17. Wang A, Johnston SC, Chou J, Dean D. A systemic network for Chlamydia pneumoniae entry into human cells. J Bacteriol. 2010;192(11):2809-15.

18. Leung AK, Young AG, Bhutkar A, Zheng GX, Bosson AD, Nielsen $C B$ Sharp PA. Genome-wide identification of Ago2 binding sites from mouse embryonic stem cells with and without mature microRNAs. Nat Struct Mol Biol. 2011;18(2):237-44

19. Chi SW, Zang JB, Mele A, Darnell RB. Argonaute HITS-CLIP decodes microRNA-mRNA interaction maps. Nature. 2009:460(7254):479-86.

20. Marson A, Levine SS, Cole MF, Frampton GM, Brambrink T, Johnstone S, Guenther MG, Johnston WK, Wernig M, Newman J, et al. Connecting microRNA genes to the core transcriptional regulatory circuitry of embryonic stem cells. Cell. 2008;134(3):521-33.

21. Maere S, Heymans K, Kuiper M. BiNGO: a Cytoscape plugin to assess overrepresentation of gene ontology categories in biological networks. Bioinformatics. 2005;21(16):3448-9.

22. Bader GD, Hogue CW. An automated method for finding molecular complexes in large protein interaction networks. BMC Bioinformatics. 2003;4:2.

23. Miyoshi N, Ishii H, Nagano H, Haraguchi N, Dewi DL, Kano Y, Nishikawa S, Tanemura M, Mimori K, Tanaka F, et al. Reprogramming of mouse and human cells to pluripotency using mature microRNAs. Cell Stem Cell. 2011;8(6):633-8

24. Kuchen S, Resch W, Yamane A, Kuo N, Li Z, Chakraborty T, Wei L, Laurence A, Yasuda T, Peng $S$, et al. Regulation of microRNA expression and abundance during lymphopoiesis. Immunity. 2010;32(6):828-39.

25. Hu G, Kim J, Xu Q, Leng Y, Orkin SH, Elledge SJ. A genome-wide RNAi screen identifies a new transcriptional module required for self-renewal. Genes Dev. 2009;23(7):837-48.

26. Holland JD, Klaus A, Garratt AN, Birchmeier W. Wnt signaling in stem and cancer stem cells. Curr Opin Cell Biol. 2013;25(2):254-64.

27. Wend P, Holland JD, Ziebold U, Birchmeier W. Wnt signaling in stem and cancer stem cells. Semin Cell Dev Biol. 2010;21(8):855-63.

28. Burdon T, Smith A, Savatier P. Signalling, cell cycle and pluripotency in embryonic stem cells. Trends Cell Biol. 2002;12(9):432-8.

29. Cho EG, Zaremba JD, McKercher SR, Talantova M, Tu S, Masliah E, Chan SF, Nakanishi N, Terskikh A, Lipton SA. MEF2C enhances dopaminergic neuron differentiation of human embryonic stem cells in a parkinsonian rat model. PLoS One. 2011:6(8), e24027.

30. Meissner A, Mikkelsen TS, Gu H, Wernig M, Hanna J, Sivachenko A, Zhang X, Bernstein BE, Nusbaum C, Jaffe DB, et al. Genome-scale DNA methylation maps of pluripotent and differentiated cells. Nature. 2008;454(7205):766-70.

31. Tay Y, Zhang J, Thomson AM, Lim B, Rigoutsos I. MicroRNAs to Nanog, Oct4 and Sox2 coding regions modulate embryonic stem cell differentiation. Nature. 2008;455(7216):1124-8.

32. Guo X, Liu Q, Wang G, Zhu S, Gao L, Hong W, Chen Y, Wu M, Liu H, Jiang C, et al. microRNA-29b is a novel mediator of Sox2 function in the regulation of somatic cell reprogramming. Cell Res. 2013;23(1):142-56.

33. Garzon R, Liu S, Fabbri M, Liu Z, Heaphy CE, Callegari E, Schwind S, Pang J, Yu J, Muthusamy N, et al. MicroRNA-29b induces global DNA hypomethylation and tumor suppressor gene reexpression in acute myeloid leukemia by targeting directly DNMT3A and $3 \mathrm{~B}$ and indirectly DNMT1. Blood. 2009;113(25):6411-8.

34. Boland MJ, Nazor KL, Loring JF. Epigenetic regulation of pluripotency and differentiation. Circ Res. 2014;115(2):311-24.

35. Wang A, Du Y, He Q, Zhou C. A quantitative system for discriminating induced pluripotent stem cells, embryonic stem cells and somatic cells. PLoS One. 2013;8(2), e56095.

36. Wang A, Huang K, Shen Y, Xue Z, Cai C, Horvath S, Fan G. Functional modules distinguish human induced pluripotent stem cells from embryonic stem cells. Stem Cells Dev. 2011;20(11):1937-50.

37. Flynn RA, Chang HY. Long noncoding RNAs in cell-fate programming and reprogramming. Cell Stem Cell. 2014;14(6):752-61.

38. Li H, Durbin R. Fast and accurate long-read alignment with Burrows-Wheeler transform. Bioinformatics. 2010;26(5):589-95.

39. Wang A, Al-Kuhlani M, Johnston SC, Ojcius DM, Chou J, Dean D: Transcription Factor Complex AP-1 Mediates Inflammation Initiated by Chlamydia pneumoniae Infection. Cell Microbiol. 2013;15(5):779-94.

\section{Submit your next manuscript to BioMed Central and take full advantage of:}

- Convenient online submission

- Thorough peer review

- No space constraints or color figure charges

- Immediate publication on acceptance

- Inclusion in PubMed, CAS, Scopus and Google Scholar

- Research which is freely available for redistribution

Submit your manuscript at www.biomedcentral.com/submit 